\title{
BROADCASTING WITH PRIORITY
}

\author{
K. V. S. PRASAD
}

\begin{abstract}
Adding priorities to CCS is difficult, and involves two-stage operational semantics or other complications. By contrast, priorities can be added very simply to a calculus of broadcasting systems (CBS). The reason is the input/output distinction made in CBS, with output actions being autonomous. Priority makes sense only for autonomous actions.

As in unprioritised CBS, both strong and weak bisimulation are congruences, and capture the intuitively desired equivalences. CBS is also a powerful and natural language, offering an interesting new programming paradigm. Several examples show that priorities extend both the power and the paradigm.
\end{abstract}

The public address system at an airport can give bomb alerts, say, priority over flight announcements. Everyone hears messages read out over the system, but individual responses vary. Priority assignment cannot depend on the actual public responses to the contending messages, even if expectations about responses play a role. Priority here is only a matter of choosing between the messages themselves.

Priorities in the marketplace are more complex, because buyer and seller must agree for a sale to take place. Sellers' minimum prices and buyers' maximum prices must be met, but further preferences for advantageous prices are not relevant unless there are competing parties to do business with. A broker arranging sales that respect everybody's priorities has a harder task than the announcer at the airport.

The handshake (or rendezvous) model of communication is similar to the market model. An indication of the difficulty of respecting priorities in this model is the "priority inversion" problem in Ada [BA90]. This model is also predominant in process calculus. The several papers [BBK85, CH88, CW91] that add priority to process calculus have all used this model, and therefore suffer from the broker's difficulties. In [CH88], an a priori semantics works out what might happen, and a second stage works out what actually happens. In [CW91], transitions are predicated on what the environment can do.

This paper adds priorities to a calculus of broadcasting systems (CBS) [Pra93c]. The resulting CBS with priorities (PCBS) is strikingly simple, and compares very favourably with CCS with priorities. This confirms the intuition above, since the

Key words and phrases. Broadcast, priority, process calculi, bisimulation, functional programming.

Author's address: Department of Computing Science, Chalmers University of Technology, S41296 Gothenburg, Sweden. E-mail: prasad@cs.chalmers.se.

Funding: From the Swedish Government agencies TFR and NUTEK, the latter supporting Chalmers' membership of the Esprit Basic Research Action CONCUR2. 
communication model of CBS resembles a public address system. It also demonstrates that there is no inherent difficulty in incorporating priorities into the mathematical apparatus of operational semantics and observational equivalences.

The ease of the transition from CBS to PCBS might lead one to doubt that anything real has been achieved. Several programming examples follow to quell such doubts. They show novel uses of priority far beyond simple interrupts. PCBS, like CBS, is a programming language as well as a process calculus. A simulator for PCBS has been implemented, and all the examples here have been run on it.

Readers are assumed to be familiar with CCS [Mil89] and with functional programming. Familiarity with [Pra93c] is not strictly necessary, but would help. More discussion of the CBS model of computation can be found in [Pra93b, Pra91], which present older versions of CBS, significantly different from that of [Pra93c].

\section{The Syntax and Semantics of PCBS}

A PCBS process of type Proc $\alpha$ says or hears values of type $\alpha$ and evolves to other processes of type Proc $\alpha$. Saying and hearing $v: \alpha$ are written $\stackrel{v ! k}{\longrightarrow}$ and $\stackrel{v ? k}{\longrightarrow}$, where $k$ is the priority of the action. Priorities are natural numbers, with 0 the highest. CBS is the special case of PCBS where $k$ is always 0 .

A useful auxiliary notion is that of the priority of a process, defined to be $k$ if it has an utterance at priority $k$. There can be only one such $k$; if a process has several components, only the most urgent will speak. A process with nothing to say is said to have priority " $\infty$ ".

In (P)CBS, whatever is said is heard by all processes in parallel with the speaker. In PCBS, hearing is read as encoded permission to speak: a process with things to say will not hear less urgent speech, which therefore cannot take place.

The syntax and semantics of PCBS are given in Table 1. Priorities are used only in the rules for translation and in the side conditions for guarded sums. The calculus abstracts away from the details of the evaluation, written $\Downarrow$, of data expressions. The data type $\alpha$ may itself involve the type Proc $\beta$ for some $\beta$. This paper does not explore this possibility, i.e., attention is restricted to first order PCBS.

The guarded sum $\sum_{i \in I} e_{i} !_{k_{i}} P_{i}+? f$ is distfix notation for a single constructor with several arguments, $e_{i}, k_{i}, P_{i}$ and $f$. NOTE: $e !_{k} P$ is not a process by itself, and there is no general binary + . The special cases ? $f$, where $I=\emptyset$, and $e !_{k} P+? f$, where $I$ is a singleton, are the only ones used in programming.

The process ? $f$ has nothing to say. It hears any utterance $v$ of the environment, no matter at what priority it is said, and becomes the process $f v$.

$$
\text { ?f } \stackrel{5 ? 3}{\longrightarrow} f 5 \quad ? \lambda x .\left((x+1) !_{3} P+? f\right) \stackrel{5 ? 2}{\longrightarrow}(5+1) !_{3} P+? f
$$

$o$ can be defined directly or derived upto $\sim$ (strong bisimulation, see Section 4 ).

$$
\circ \stackrel{v ? k}{\longrightarrow} 0 \quad \quad 0 \sim X \text { where } X \stackrel{\text { def }}{=} ? \lambda x . X
$$

Only closed processes communicate, not abstractions like $\lambda x .(x+1) !_{3} P$ or expressions with free data variables like $(x+1) !_{3} P$. The process $(5+1) !_{3} P+? f$ has priority 3 ; it wishes to say 6 at priority 3 and become $P$. If it hears $v$, which has to be said at priority 3 or higher, it evolves to $f v$.

$$
(5+1) !_{3} P+? f \stackrel{6 ! 3}{\longrightarrow} P \quad(5+1) !_{3} P+? f \stackrel{4 ? k}{\longrightarrow} f 4 \text { if } k \leq 3
$$


Let $\alpha$ be a datatype. Let $\tau$ be a distinguished value, and $\alpha_{\tau}$ the disjoint union of $\alpha$ and $\{\tau\}$.

Let $P, P_{i}:$ Proc $\alpha$, the type of processes communicating $\alpha$ 's. Let $I$ be a finite set. Let $e_{i}: \alpha_{\tau}$, $k_{i}: \mathbb{N}$ and $f: \alpha \rightarrow$ Proc $\alpha$.

Let $P_{\beta}:$ Proc $\beta$. Let $\phi=\left\langle k_{\phi}, \phi^{\dagger}, \phi_{1}\right\rangle$, where $k_{\phi}: \mathbb{N}, \phi^{\dagger}: \beta_{\tau} \rightarrow \alpha_{\tau}$ and $\phi_{\perp}: \alpha_{\tau} \rightarrow \beta_{\tau}$ satisfying $\phi^{\dagger} \tau=\tau$ and $\phi_{1} \tau=\tau$.

Let $b$ : Bool, and let $A$ range over constants, declared in (mutually) recursive guarded definitions $A \stackrel{\text { def }}{=} P$. Then the elements of Proc $\alpha$ are given by

$$
P::=\sum_{i \in I} e_{i} !_{k_{i}} P_{i}+? f|P| P\left|\phi P_{\beta}\right| \text { if } b \text { then } P \text { else } P \mid A
$$

$v: \alpha, w: \alpha_{\tau}$ and $u: \beta_{\tau}$ are canonical values. The relation $\downarrow$ means "can evaluate to". $\stackrel{w ! k}{\longrightarrow}$ and $\stackrel{w ? k}{\longrightarrow}$ are actions at priority $k$, where $k: \mathbb{N}$. Let $P^{\prime}, P_{i}^{\prime}: \operatorname{Proc} \alpha$; and $P_{\beta}^{\prime}: \operatorname{Proc} \beta$.

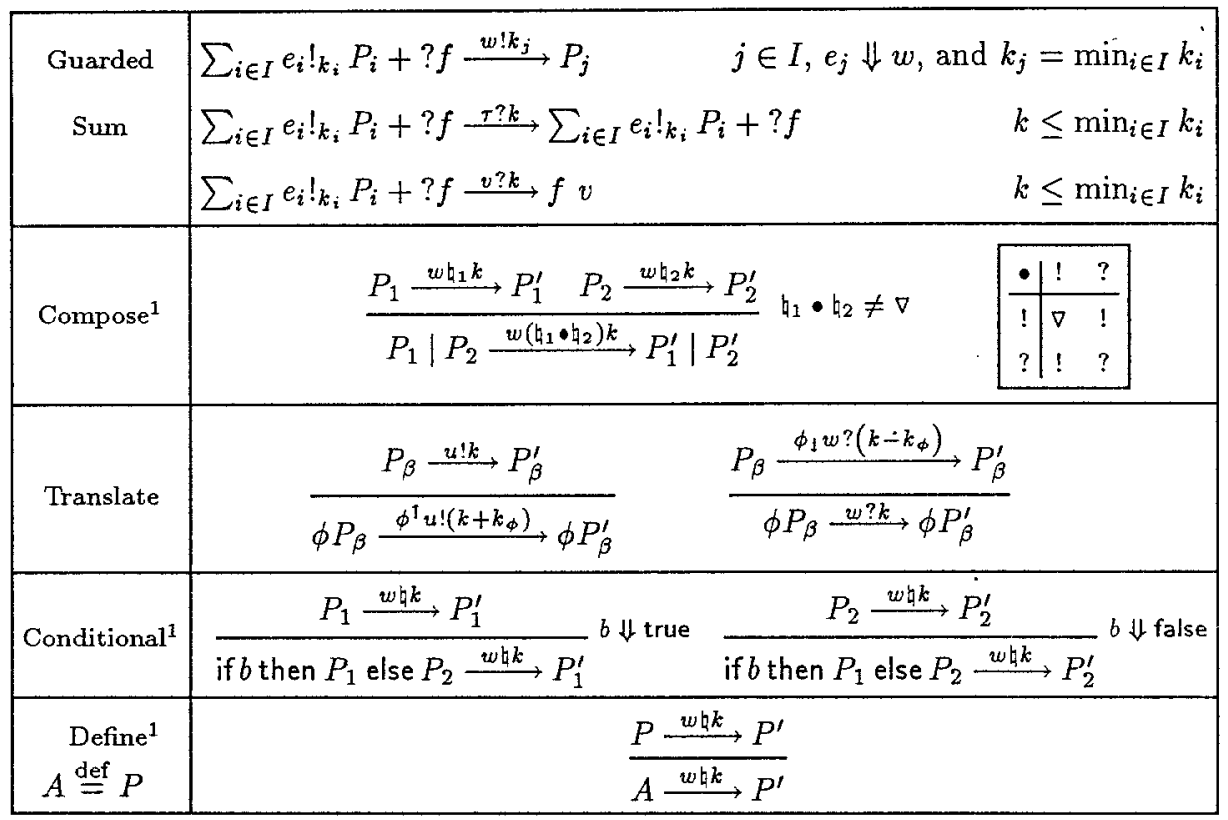

$1 h, h_{1}, b_{2}$ range over $\{!, ?\} . \nabla$ means "undefined" in the synchronisation algebra for $\bullet$

TABLE 1. The syntax of PCBS and the semantics of closed processes

A special case of $e !_{k} P+? f$ is important. Let

$$
e ! !_{k} P \stackrel{\text { def }}{=} e !_{k} P+? \lambda x .\left(e !_{k} P\right)
$$

Abusing notation, $e !_{k} P$ is written $e !_{k} P$. Thus " $e !_{k} P$ " outside a + context stands for " $e ! !_{k} P$ ". Suppose $e \Downarrow v$, i.e., $e$ evaluates to $v$. The process $e !_{3} P$ wishes to say $v$ at priority 3 ; it hears, but ignores, anything at priority 3 or lower.

$$
e !_{k} P \stackrel{v ! k}{\longrightarrow} P \text { if } e \Downarrow v \quad \quad e !_{k} P \stackrel{v ? k^{\prime}}{\longrightarrow} e !_{k} P \text { if } k^{\prime} \leq k
$$

If $e !_{k} P$ is defined by these rules and $e !_{k} P$ by recursion, then $e !_{k} P \sim e ! !_{k} P$.

Communication is synchronous. At every step, one of the processes in a parallel composition says something, and all the other processes hear it. Speakers go one 
at a time, and are independent of the number of listeners. $\mid$ is associative.

$$
\begin{aligned}
& ? f\left|5 !_{3} P\right| ? \lambda y \cdot y !_{1} \circ \stackrel{5 !_{3}}{\longrightarrow} f 5|\quad P| 5 !_{1} \circ \\
& ? f\left|5 !_{3} P\right| ? \lambda y \cdot y !_{1} \circ \stackrel{4 ? 2}{\longrightarrow} f 4\left|5 !_{3} P\right| 4 !_{1} \circ
\end{aligned}
$$

All processes including parallel compositions respond deterministically to what they hear. The only guarded sums used in programming, ? $f$ and $v !_{k} P+? f$, are deterministic also for speech. Contending speakers in parallel are in fact the only source of non-determinism. To permit an expansion theorem, a more general guarded sum of the form $\sum_{i \in I} e_{i} !_{k_{i}} P_{i}+? f$ is needed. Only finite parallel compositions are used, so $I$ can be restricted to be a finite set.

$$
\begin{aligned}
2 !_{1} P \mid 7 !_{2} Q & \sim 2 !_{1}\left(P \mid 7 !_{2} Q\right)+7 !_{2}\left(2 !_{1} P \mid Q\right)+2 \lambda x .\left(2 !_{1} P \mid 7 !_{2} Q\right) \\
& \sim 2 !_{1}\left(P \mid 7 !_{2} Q\right) \\
& +? \lambda x .\left(2 !_{1} P \mid 7 !_{2} Q\right)
\end{aligned}
$$

The second line follows because outranked output guards can never be used. The process $\sum_{i \in I} e_{i} !_{k_{i}} P_{i}+? f$ has priority $\min _{i \in I} k_{i}$. It non-deterministically says one of the most urgent things it has to say, except if preempted by the environment.

$$
\begin{aligned}
& \sum_{i \in I} e_{i} !_{k_{i}} P_{i}+? f \stackrel{5 ? k}{\longrightarrow} f 5 \quad k \leq \min _{i \in I} k_{i} \\
& \sum_{i \in I} e_{i} !_{k_{i}} P_{i}+? f \stackrel{v ! k_{j}}{\longrightarrow} P_{j} \quad j \in I, e_{j} \Downarrow v \text {, and } k_{j}=\min _{i \in I} k_{i}
\end{aligned}
$$

Finally, a translator $\phi$ is a triple $\left\langle k_{\phi}, \phi^{\uparrow}, \phi_{\downarrow}\right\rangle$. The process $\phi P$ says $\phi^{\uparrow} 5$ at priority $k+k_{\phi}$ if $P$ says 5 at $k$, and hears 4 at $k$ if $P$ hears $\phi_{\downarrow} 4$ at $k-k_{\phi}$. Here $n-m$ is $n-m$ if $n \geq m$ and 0 otherwise. Thus a translator can deprioritise a subsystem.

Translators interface between systems of different types. Hiding and restriction are achieved by translation to $\tau$, an aside appended to every data type. Asides can be spoken but not heard. In implementations of PCBS, the premise $P \stackrel{\tau ! k}{\longrightarrow} P^{\prime}$ yields the conclusion $P\left|Q \stackrel{\tau ! k}{\longrightarrow} P^{\prime}\right| Q$ provided $k \leq \pi(Q)$. This is equivalent to the more concise formulation here, where asides are heard but always ignored:

$$
Q \stackrel{\tau ? k}{\longrightarrow} Q^{\prime} \text { where } Q^{\prime} \equiv Q \text { up to unfolding of constants and conditionals }
$$

Translating functions must map $\tau$ to $\tau$.

\section{Properties of the transition System}

DeFinition 1. The priority $\pi(P)$ of a closed process $P$ is given by the rules

$$
\begin{array}{ll}
\pi(? f) & =\infty \\
\pi\left(\sum_{i \in I} e_{i} !_{k_{i}} P_{i}+? f\right) & =\min _{i \in I} k_{i} \text { if } I \neq \emptyset \\
\pi(P \mid Q) & =\min (\pi(P), \pi(Q)) \\
\pi\left(\left\langle k_{\phi}, \phi^{\uparrow}, \phi_{\downarrow}\right\rangle P\right) & =k_{\phi}+\pi(P) \\
\pi\left(\text { if } b \text { then } P_{1} \text { else } P_{2}\right) & =\text { if } b \text { then } \pi\left(P_{1}\right) \text { else } \pi\left(P_{2}\right) \\
\pi(A) & =\pi(F) \quad \text { where } A \stackrel{\text { def }}{=} F
\end{array}
$$

Let $P \stackrel{w \natural k}{\longrightarrow}$ abbreviate "there exists $P^{\prime}$ such that $P \stackrel{w \natural k}{\longrightarrow} P^{\prime \prime}$, and let $P \stackrel{w \emptyset k}{\longrightarrow}$ abbreviate "there is no $P^{\prime}$ such that $P \stackrel{w \sharp k}{\longrightarrow} P^{\prime \prime}$ ".

Proposition 2. $\pi(P)=k$ and $k \neq \infty$ iff $\exists v, P^{\prime}$ such that $P \stackrel{v ! k}{\longrightarrow}$. Also, $P \stackrel{v ! k}{\longrightarrow}$ and $P \stackrel{v^{\prime} ! k^{\prime}}{\longrightarrow}$ implies $k=k^{\prime}$. So $P \stackrel{w ! k}{\longrightarrow}$ if $k<\pi(P)$. 
REMARK 3. For all $P$, the set $\{\langle w, k\rangle \mid P \stackrel{w ! k}{\longrightarrow}\}$ is finite. If priorities were to increase from 0 upwards, this fact would be needed to ensure that priority was well defined, for a process like $\sum_{i \in \mathbb{N}} e_{i} !_{i} P_{i}+? f$ would stop all progress in any system of which it is a component. (A "priority stop" process).

Proposition 4. $P \stackrel{w ? k}{\longrightarrow}$ iff $k \leq \pi(P)$.

Proposition 5. $\forall P, w, \exists ! P^{\prime}$ such that $P \stackrel{w ? 0}{\longrightarrow} P^{\prime}$.

Definition 6. $P / w$, the image of $P$ under $w$, is the $P^{\prime}$ such that $P \stackrel{w ? 0}{\longrightarrow} P^{\prime}$.

Proposition $7 . k \leq \pi(P)$ implies $P \stackrel{\tau ? k}{\longrightarrow} P^{\prime}$, where $P \sim P^{\prime}$.

In fact, if $k \leq \pi(P)$ then $P \stackrel{r ? k}{\longrightarrow} P^{\prime}$, where $P \equiv P^{\prime}$ up to unfolding of constants and conditionals. So $(? f) / v=f v$ and $P / \tau \sim P$. Section 4 defines $\sim$.

\section{From CBS to PCBS}

It is instructive to consider alternative designs for PCBS, starting from the same syntax, and the same interpretation: $3 !_{1} A$ wishes to say " 3 " at priority 1 . A first attempt could start from Definition 1 , which is purely syntactic. The parallel rule could then be taken to be $P \stackrel{v !}{\longrightarrow} P^{\prime}$ and $Q \stackrel{v ?}{\longrightarrow} Q^{\prime}$ and $\pi(P) \leq \pi(Q)$ imply $P\left|Q \stackrel{v !}{\longrightarrow} P^{\prime}\right| Q^{\prime}$. It allows $3 !_{1} A\left|4 !_{2} B \stackrel{3 !}{\longrightarrow} A\right| 4 !_{2} B$ to be derived, but not $3 !_{1} A\left|4 !_{2} B \stackrel{4 !}{\longrightarrow} 3 !_{1} A\right| B$.

But in this calculus $P \sim R$ does not imply $P|Q \sim R| Q$. For $3 !_{1} A \sim 3 !_{2} A$, yet $3 !_{1} A \mid 5 !_{1} C$ can say " 3 ", while $3 !_{2} A \mid 5 !_{1} C$ cannot. To repair this, annotate speech with the speaker's priority: $3 !_{1} A \stackrel{3 ! 1}{\longrightarrow} A$. Now $3 !_{1} A \not 3 !_{2} A$. The annotation is only to help reasoning; it is not part of what is said. The announcer reads out the chosen message, not (usually) why it was chosen over others.

Proposition 2 holds for this intermediate calculus. The side-condition $\pi(P) \leq$ $\pi(Q)$ is equivalent to $Q \frac{w ! k / f}{\text { if }} k<\pi(P)$. Despite this negative premise, the transition system is well defined, for the definition of $\pi(P)$ is independent of the transitions of $P$.

The final step to PCBS, that of annotating hearing with the priority of the speech heard, is less important than the annotation of speech. It encodes process priority into hearing transitions, and therefore absorbs the side-condition of the parallel rule into the second premise. Proposition 4 describes the new annotation.

\section{Strong Bisimulation}

Let $\mathbb{P}_{c l}$ be the set of closed processes. Let $\downarrow \in\{!, ?\}$.

Definition 8 (Strong bisimulation for closed processes). $\mathcal{R} \subseteq \mathbb{P}_{c l} \times \mathbb{P}_{c l}^{k}$ is a strong bisimulation if whenever $P \mathcal{R} Q$,

(i) if $P \stackrel{w h k}{\longrightarrow} P^{\prime}$ then $\exists Q^{\prime}$ such that $Q \stackrel{w h^{\prime} k}{\longrightarrow} Q^{\prime}$ and $P^{\prime} \mathcal{R} Q^{\prime}$,

(ii) if $Q \stackrel{w \natural k}{\longrightarrow} Q^{\prime}$ then $\exists P^{\prime}$ such that $P \stackrel{w \natural k}{\longrightarrow} P^{\prime}$ and $P^{\prime} \mathcal{R} Q^{\prime}$

The largest strong bisimulation is an equivalence, denoted $\sim$. It can be extended to process abstractions thus: $f \sim g$ iff $\forall v, f v \sim g v$. So $f \sim g$ implies ?f ?g.

Proposition 9. $\sim$ is a congruence for $C B S$. 
Definition 10. For any $\mathbb{L} \subseteq \alpha$, if the transmissions $w ! k$ of $P$ and all its derivatives are such that $w \in \mathbb{L} \cup\{\tau\}$ then $P$ has sort $\mathbb{L}$, written $P: \mathbb{L}$.

In $\sum_{i \in I} e_{i} !_{k_{i}} P_{i}+? f$, the set $I$ is finite. If we also assume a standard order for its elements, the "sum" above is just a list. Laws 1(a) and 1(b) below say that this list is a set, and justify the sum notation. Laws $1(\mathrm{c}), 3(\mathrm{a}), 3(\mathrm{c})$ and 4 below distinguish prioritised CBS from ordinary CBS. In Law 2, $\mathbb{P}$ is the set of all processes.

Proposition 11 (Strong bisimulation laws).

(1) (a) $\ldots+e_{i} !_{k_{i}} P_{i}+e_{j} !_{k_{j}} P_{j}+\ldots+? f \sim \ldots+e_{j} !_{k_{j}} P_{j}+e_{i} !_{k_{i}} P_{i}+\ldots+? f$

(b) $\ldots+e_{i} !_{k_{i}} P_{i}+e_{i} !_{k_{i}} P_{i}+\ldots+? f \sim \ldots+e_{i} !_{k_{i}} P_{i}+\ldots+? f$

(c) $\ldots+e_{i} !_{k_{i}} P_{i}+e_{j} !_{k_{j}} P_{j}+\ldots+? f \sim \ldots+e_{i} !_{k_{i}} P_{i}+\ldots+$ ?f if $k_{i}<k_{j}$

(2) $(\mathbb{P} / \sim, 1,0)$ is a commutative monoid.

(3) (a) $\phi\left(e !_{k} P\right) \sim\left(\phi^{\uparrow} e\right) !_{k+k_{\phi}} \phi P$

(b) $\phi(? f) \sim X$ where $X \stackrel{\text { def }}{=}$ ? $\lambda x$. if $\phi_{\downarrow} x=\tau$ then $X$ else $\phi\left(f\left(\phi_{\downarrow} x\right)\right)$

(c) $\phi\left(\sum_{i \in I} e_{i} !_{k_{i}} P_{i}+? f\right) \sim X$ where

$X \stackrel{\text { def }}{=} \sum_{i \in I}\left(\phi^{\dagger} e_{i}\right) !_{k_{i}+k_{\phi}}\left(\phi P_{i}\right)+$ ? $\lambda x$. if $\phi_{\downarrow} x=\tau$ then $X$ else $\phi\left(f\left(\phi_{\downarrow} x\right)\right)$

(4) $\phi \psi P \sim \phi \circ \psi P$ where $\phi \circ \psi=\left\langle k_{\phi}+k_{\psi}, \phi^{\uparrow} \circ \psi^{\uparrow}, \psi_{\downarrow} \circ \phi_{\downarrow}\right\rangle$.

(5) $\phi\left(P_{1} \mid P_{2}\right) \sim \phi P_{1} \mid \phi P_{2}$ if $\forall v \in \mathbb{L}_{1} \cup \mathbb{L}_{2}, \phi_{\downarrow} \phi^{\uparrow} v=v$, where $P_{1}: \mathbb{L}_{1}$ and $P_{2}: \mathbb{L}_{2}$

The expansion theorem below does not need to take care of priorities. Law 1(c) applied to the r.h.s. shows that all is well.

Proposition 12 (Expansion Theorem). Let $r \in\{0,1\}$. Then

$$
P_{0} \mid P_{1} \sim \sum_{r, w, k} w !_{k}\left(P_{r}^{\prime} \mid P_{1-r} / w\right)+? \lambda x .\left(P_{0} / x \mid P_{1} / x\right) \text { where } P_{r} \stackrel{w ! k}{\longrightarrow} P_{r}^{\prime} .
$$

$\mathrm{CBS}^{+}$, the calculus of [Pra93b], is an unprioritised calculus with ? and ! prefixes, 0 and + as primitive constructors. The equations 1(a) and 1(b) of Proposition 11 together with the expansion theorem constitute a complete axiom system for finite processes of $\mathrm{CBS}^{+}$. This result has not yet been adapted to the CBS of [Pra93c]. PCBS was earlier built on $\mathrm{CBS}^{+}$, and it was then clear that $1(\mathrm{c})$ was the only law that distinguished the two. It seems a reasonable conjecture that this is the case in the present calculus as well.

Definition 13 (Priority abstracted bisimulation For Closed PRocesses). $\mathcal{R} \subseteq \mathbb{P}_{c l} \times \mathbb{P}_{c l}$ is a priority abstracted bisimulation if whenever $P \mathcal{R} Q$,

(i) if $P \stackrel{w \natural k}{\longrightarrow} P^{\prime}$ then $\exists Q^{\prime}, k^{\prime}$ such that $Q \stackrel{w \natural k^{\prime}}{\longrightarrow} Q^{\prime}$ and $P^{\prime} \mathcal{R} Q^{\prime}$,

(ii) if $Q \stackrel{w \boxminus k}{\longrightarrow} Q^{\prime}$ then $\exists P^{\prime}, k^{\prime}$ such that $P \stackrel{w \downarrow k^{\prime}}{\longrightarrow} P^{\prime}$ and $P^{\prime} \mathcal{R} Q^{\prime} \square$

The largest priority abstracted bisimulation is an equivalence, denoted $\simeq$. This is not a congruence, as Section 4 pointed out, for $3 !_{1} A \simeq 3 !_{2} A$, yet $3 !_{1} A \mid 5 !_{1} C \stackrel{3 ! 1}{\longrightarrow}$, while $3 !_{2} A \mid 5 !_{1} C \stackrel{3 ! k}{f}$ for any $k$. Let $\simeq{ }^{c}$ be the largest congruence contained in $\simeq$.

Proposition 14. $\sim=\simeq^{c}$

Proof. It is easy to see that $\sim \subseteq \simeq$, and $\sim$ is a congruence. For the other direction, let $P \simeq^{c} Q$, and let $P \stackrel{w_{q} k}{\longrightarrow} P^{\prime}$. Then $Q \stackrel{w h k^{\prime}}{\longrightarrow} Q^{\prime}$, and $P^{\prime} \simeq^{c} Q^{\prime}$. But $k^{\prime}=k$, otherwise a $\mid$ context can be found that can distinguish $P$ and $Q$. 


\section{Weak Bisimulation}

For convenience, let $P \stackrel{r !}{\longrightarrow} P^{\prime}$ stand for $\exists k$ such that $P \stackrel{\tau ! k}{\longrightarrow} P^{\prime}$.

Definition 15 (WEAK Bisimulation FOR ClOSEd PROCESSES). $\mathcal{R} \subseteq \mathbb{P}_{c l} \times \mathbb{P}_{c l}$ is a weak bisimulation if whenever $P \mathcal{R} Q$,

(i) if $P \stackrel{w h k}{\longrightarrow} P^{\prime}$ then $\exists Q^{\prime}$ such that $Q \stackrel{\tau !^{*} w h k \tau !^{*}}{\longrightarrow} Q^{\prime}$ and $P^{\prime} \mathcal{R} Q^{\prime}$, (ii) if $Q \stackrel{w \curvearrowleft k}{\longrightarrow} Q^{\prime}$ then $\exists P^{\prime}$ such that $P \stackrel{\tau^{*} w \llbracket k \pi !^{*}}{\longrightarrow} P^{\prime}$ and $P^{\prime} \mathcal{R} Q^{\prime}$

The largest weak bisimulation is an equivalence, denoted $\approx$.

Bisimulation here is formally similar to its CCS counterpart, but the effects are different. As with CBS, $\tau !_{k} P \approx P$ in general! Let $P \stackrel{\text { def }}{=} ? \lambda x . x !_{2} \circ$ and $Q \stackrel{\text { def }}{=} \tau !_{3} P$. Then $P \not \approx Q$, since $P$ will always echo its input, but $Q$ may fail to do so: $Q \stackrel{5 ? 2}{\longrightarrow} Q$. This cannot be matched by $P$ since it has to receive, and become 5 ! o $\approx Q$.

Also, $\tau !_{k}, v !_{k} P \approx v !_{k} P$ only if $k^{\prime} \leq k$. If $k^{\prime}>k$ the left hand process will tolerate a transmission of priority $k^{\prime}$, but the right won't. Earlier definitions of bisimulation for PCBS prescribed that $\tau$ s preceding the matching action had to be of equal or higher priority. Interestingly, the apparently more liberal definition above captures the same effect. [Pra93b] devises tests to tell unequal processes apart, and suggests that $\approx$ is therefore a meaningful equivalence for CBS. The same argument can be made for PCBS. Because sums are guarded, the following pleasant property holds.

Proposition $16 . \approx$ is a congruence for PCBS.

\section{VALUE PRIORITY}

A very similar calculus results if a fixed priority function $\zeta: \alpha \rightarrow \mathbb{N}$ is associated with the transmitted data type. Translation functions can now alter the priorities of actions. For the calculus to be well behaved, it is sufficient that translation should preserve the priority order strictly (i.e., be monotonic, and maintain inequalities), and that $\zeta\left(\phi_{\downarrow} \phi^{\uparrow} v\right)=\zeta v$. This confirms that the deprioritisation operator of PCBS, which seems anyway adequate, is as powerful as it can be.

\section{CCS WITH PRIORITIES}

The difficulties in adding priorities to CCS can be traced to the fact that handshake communication makes an autonomous action out of two controlled ones.

[CH88] begins with an a priori semantics which labels transitions with actions and their priorities. The second stage then says that prioritised actions are unconstrained, but unprioritised actions can only take place if they are not preempted by prioritised $\tau$ 's - the negative premise. The resulting new axiom is a. $P+\tau^{\prime} . Q \sim \tau^{\prime} . Q$ where $\tau^{\prime}$ is a prioritised $\tau$, the cognate of Law 1(c) of Proposition 11. But the result is not entirely satisfactory even at the cost of a two stage operational semantics, for $a . P+b . Q \nsim b . Q$ if $b$ is of higher priority than $a$-the basic difficulty mentioned in the introduction. The authors of [CH88] say that such a possibility would be useful; they also point out that then such actions cannot be restricted! This is precisely the scenario of PCBS.

Defining weak bisimulation for this model is non-trivial [CC93]; it is done by abstracting $\tau \mathrm{s}$ from sequences of actions in a priority sensitive way. (Remember that $\tau$ s preceding a matching action in PCBS have to be of equal or higher priority). 


\begin{tabular}{|c|c|}
\hline Programming notation & Calculus notation \\
\hline *a & $\alpha$ \\
\hline $\begin{array}{l}\text { Option } * a=\text { None }+ \text { Some } * a \\
\text { say } P t=\operatorname{Resp}(\pi, k) P^{\prime} \text { for some } t .\end{array}$ & $\begin{array}{l}\text { If } w \in \alpha_{\tau} \text { then } w=\tau \text { or } w=v \in \alpha \\
P \stackrel{w ! k}{\longrightarrow} P^{\prime}\end{array}$ \\
\hline say $P t=$ No_Resp for all $t$ & $\beta w, k, P^{\prime} . P \stackrel{w ! k}{\longrightarrow} P^{\prime}$ \\
\hline & \\
\hline $\operatorname{SAY}(e, k) P$ & $e !_{k} P$ \\
\hline LISTEN $f$. & $? f$ \\
\hline TALK $(e, k) P f$ & $e !_{k} P+? f$ \\
\hline PAR P_1 P_2 & $P_{.1} \mid P_{2}$ \\
\hline PARS $\left[P_{-} 1 ; \ldots ; P_{-}\right.$n] & $P_{1}|\ldots| P_{n}$ \\
\hline TRANS $(k, g ; h) P$ & $\phi P$ where $k_{\phi}=k, \phi^{\uparrow}=g$ and $\phi_{\downarrow}=h$ \\
\hline SAYS $k\left[e_{-} 1 ; e_{-} 2 ; \ldots\right] P$ & $e_{1} !_{k}\left(e_{2} !_{k}(\ldots P)\right)$ \\
\hline $\operatorname{BOX}(g, h) \quad\left[P_{-} 1 ; \ldots ; P_{-} n\right]$ & $\phi\left(P_{1}|\ldots| P_{n}\right)$ where $\phi^{\uparrow}=g$ and $\phi_{1}=h$ \\
\hline
\end{tabular}

TABLE 2. Correspondence between programming and calculus notation

[CW91] presents a priority sum $+^{\prime}$ similar to Occam's PRIALT; a. $P+^{\prime} b . Q$ can perform a $b$ only if the environment will not do $\bar{a}$. Now it is not clear whether $\left(\left(a . P+^{\prime} \bar{b} . Q\right) \mid\left(b . R+^{\prime} \bar{a} . S\right)\right) \backslash\{a, b\}$ can perform a $\tau$. Actions are therefore separated into input and output and the initial actions of a prisum are required to be inputs. This breaks the symmetry of pure CCS, and complicates the syntax.

The semantics is complicated: the transition relation is parameterised by the output actions the environment can do, as is the definition of bisimulation. The expected negative premise for $P+^{\prime} Q$ turns up as a side condition that the actions $P$ can accept, computed independently of the transition system, should not be offered by the environment. Lastly, a ready function is needed to adjust the environment parameter upon communication in a parallel composition.

It is almost as though [CW91] declared output actions autonomous, input actions controlled, and dealt semantically with what could (autonomously) happen. Unfortunately for this interpretation, output actions can be restricted. Worse, $\tau$ is independent of the environment as are output actions, but is classified as input, since it can be an initial action in a prisum! Since [CW91] deals with process priority rather than action priority, comparison with PCBS cannot be exact. But it does appear that the complexity of [CW91] comes from the handshake model.

\section{8, A PCBS Simulator in Lazy ML}

Table 2 shows the correspondence between CBS notation and the Lazy ML (LML for short) programming notation [AJ93]. Types and constructors are capitalised, while processes and process constructors are entirely in upper case.

The process type, Proc *a, is given in Table 3. Recursion and conditionals are not part of it. These are taken from LML. The constructor TRANS uses an existential type *b that does not occur on the left hand side. The general guarded sum is not needed for programming, only TALK.

To run a process, apply say to it. Basically, say $(\operatorname{SAY}(3,1) P)=\operatorname{Resp}(3,1) P$, and say (IISTEN $f$ ) = No_Resp. But if $R \stackrel{\text { def }}{=} 3 !_{0} P \mid 5 !_{0} Q$ then

$$
R \stackrel{3 ! 0}{\longrightarrow} P\left|5 !_{0} Q \quad R \stackrel{5 ! 0}{\longrightarrow} 3 !_{0} P\right| Q
$$




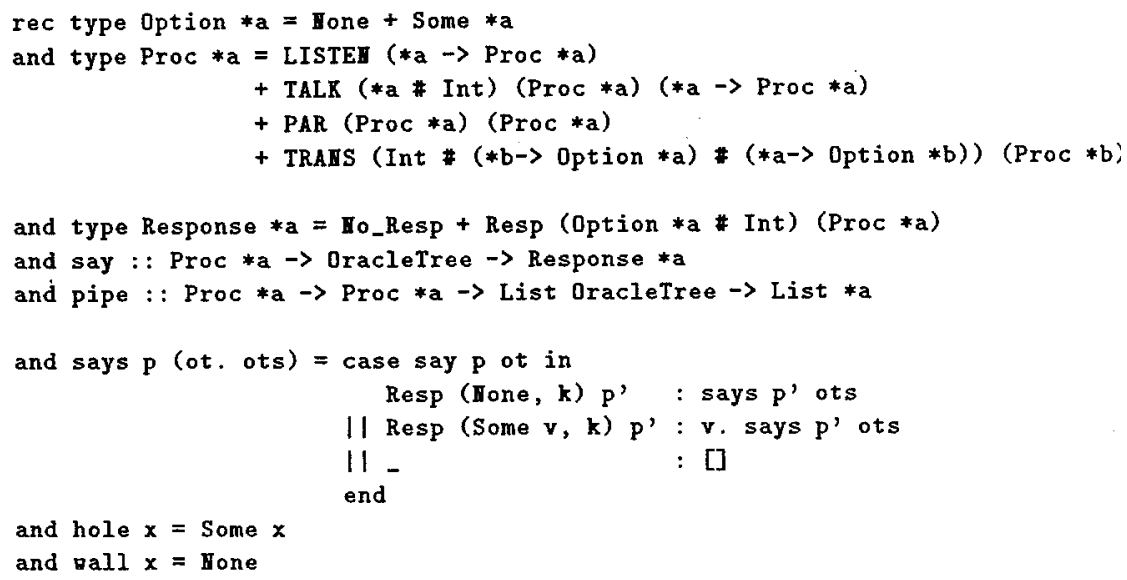

TABLE 3. The user interface of the simulator and some abbreviations

Clearly, say has to be nondeterministic. One way [Bur88] to achieve nondeterminism with functions is to put the nondeterminism in the data. say is given an extra boolean argument, an oracle, whose value will be determined at run time, but once fixed will not change. The oracle chooses between the parallel components. Thus for some oracles $t$ and $t$ ', we have

$$
\begin{aligned}
& \text { say } R t=\operatorname{Resp}(3,0)(\operatorname{PAR} P(\operatorname{SAY}(5,0) Q)) \text { and } \\
& \text { say } R t^{\prime}=\operatorname{Resp}(5,0)(\operatorname{PAR}(\operatorname{SAY}(3,0) P) Q) .
\end{aligned}
$$

say uses a tree of oracles rather than a single oracle, because after choosing the right branch in $P \mid(Q \mid R)$, a further choice has to be made.

say is usually used packaged up as says, also in Table 3. An infix "." is LML notation for "cons". says produces one trace of transmissions from the process, filtering out $\tau$ 's, and using a list of oracletrees to resolve choices along the way. It can return a finite list if the process has an evolution to one with no transmissions.

The most common interface function in this paper is pipe $p t$ which puts $p$ and $t$ in communication with one another, but returns only whatever $p$ says. It is as though the user were in the same room as $p$ when it is on the phone to $t$. In applications, $t$ can be the input or the insides of a system, and $\mathrm{p}$ the output or front end. It is implemented as says $\phi\left(\phi_{p} p \mid \phi_{t} t\right)$ with simple translators, and frequently allows a simple data type when says would need separate constructors to distinguish p's utterances from t's.

The simulator is ultimately intended to run on a parallel implementation of LML, where the first process to speak is chosen. Then the process 3 ! $0 \mid 1$ ! o will always produce 3 before looping. For now, the list of oracletrees is generated either by randtrees $r$, where $r$ is the seed for a random number generator, or by firsttrees where the choice is always the left component. The latter is useful if the system is essentially deterministic. These pseudo-oracles might make the wrong choice with 3 ! $0 \mid \perp$ ! o and produce no output at all. 


\section{Programming eXamples}

Let $I$ be a set of numbers. Then $\prod_{i \in I} i !_{i}$ o sorts $I$. The number of priority levels this program needs depends on $I$. Hardware implementations typically provide only a limited number of priority levels, so the programs that follow use only a bounded number of them, independent of the input.

Two similar disciplines that are followed both here and in [Pra93c] are that the memory needed by any process, and the size of the transmitted values, are both independent of the input data (counting integers as one "word", and making other such traditional assumptions). This allows the number of messages to be used as a measure of time taken, and the number of processes as a measure of space.

Neither complexity nor correctness are dealt with in these examples; their purpose is to demonstrate the power of the language. Some proofs were sketched in [Pra93b, Pra93c] and it is clear that the usual techniques of process calculus apply. The examples all use value passing, and the formal integration of proofs about processes with proofs about data is still being worked out.

EXAMPLE 17. [Atomic sequences; last element of a list]

Here is a simple indication that PCBS is strictly more powerful than CBS. Pure CBS, where the data type is the singleton set, is essentially useless: there is no obvious way to get information in and out of systems. Unary coding is not possible because silence cannot be detected. But pure PCBS is useful, because silence at level 0 can be detected by successful transmission at level 1 . Thus data can be transmitted in atomic sequences at level 0 , with level 1 used as an end marker.

rec $\operatorname{TRAP} n=\operatorname{TALK}(n, 1) \cdot \mathbb{I L}(\backslash x \cdot \operatorname{TRAP} x)$

and last $1=$ pipe (LISTEH ( $\backslash x$. TRAP $x$ )) (SAYS 01 NIL) firsttrees

last returns [] if 1 is empty, and a singleton list with the last element of 1 otherwise. This cannot be done within CBS. However, says and pipe can detect termination (in this and other simple cases), signalling it by returning a finite list. See [Pra93c] for examples of use of this kind of termination detection.

EXAMPLE 18. [TALK is an interrupt operator]

The motivating example from [CH88] and [CW91] is a counter that accepts "Up" and "Down" commands until interrupted. Here is a variation, a clock that counts off intervals until interrupted by any signal at priority 0 or 1 .

$$
\text { rec CLOCX } n=\operatorname{TALK}(n, 1)(\operatorname{CLOCK}(n+1))(\backslash x \text {. IIL) }
$$

To be sure to stop the clock, the signal from outside must be sent at priority 0 .

EXAMPLE 19. [Primes in increasing order: two processes]

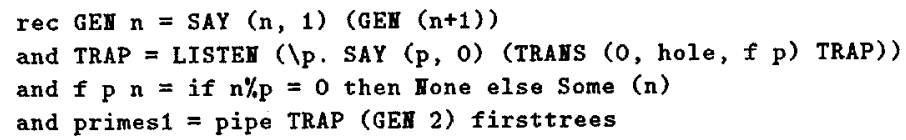

TRAP hears only primes. For each prime, it wears a further translation layer to hide all multiples of this number. Without priorities, GEN would have to wait to hear from TRAP before announcing the next number, and TRAP must make an announcement for every number, so a type Composite + Prime Int would be needed. 
EXAMPLE 20. [Primes in increasing order: new process per prime]

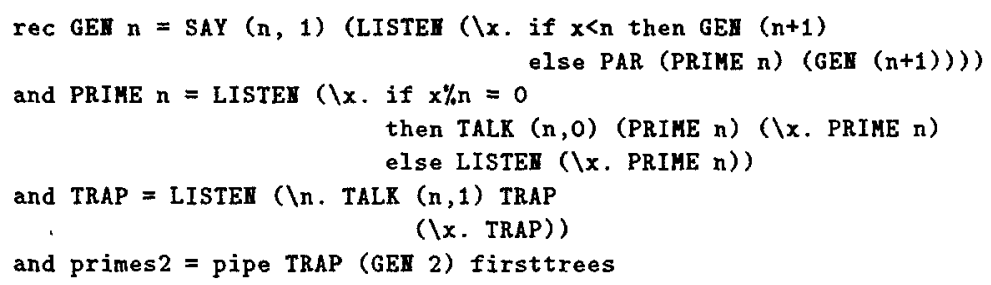

TRAP optimistically tries to declare every number to be a prime. On composite numbers, one of the prime divisors preempts it and the other divisors. GEN can tell primes from composites because the former are echoes of what it said. There is no obvious unprioritised solution corresponding to this one.

EXAmple 21. [Primes up to $n$ in increasing order: $n+1$ processes] This program lists primes in the range [2..n], by systematically testing each $p$ for divisibility by all numbers less than itself. Without priorities, the primes will not come out in order. For the same price, a faster program can be had by reversing the priorities and replacing $i * i>$ by $i=$ in the tests.

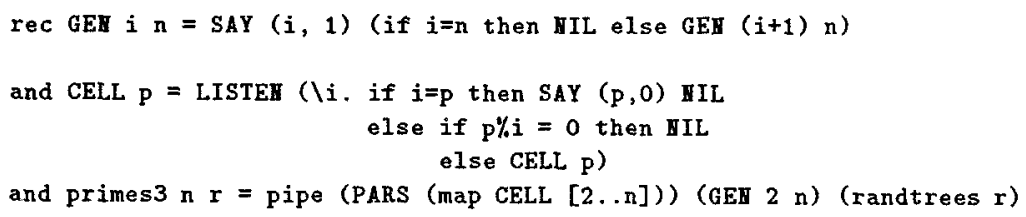

EXAMPLE 22. [Primes up to $n$ : possibly only two broadcasts per prime]

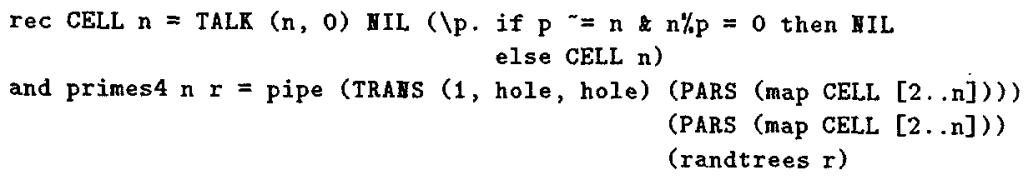

This is a two phase algorithm. In the first phase, all primes and some of the composite numbers on the far side of the pipe announce themselves. Numbers die when they hear a divisor. Survivors on the near side announce themselves in the second phase. The oracles decide which composites speak in the first phase, and in what order the primes speak in the second.

A data type could be used to tell first phase transmissions from second. The first are to be hidden. The code here uses pipe instead to get away with processes of type Int. The penalty is that there are twice as many processes.

The translator allows CELL to be reused, instead of a new process that speaks at priority 1 . The deprioritisation has the further advantage that competing processes have priority 0 also in the second phase (inside the translator). The arbitrator has to choose some speaker of highest priority. It helps if this is 0 , for then it may have to look no further. If the second phase CELLs each spoke at level 2, the arbitrator would have to check them all to make sure no one wishes to speak at 1 or 0 . 
EXAMPLE 23. [Fibonacci numbers]

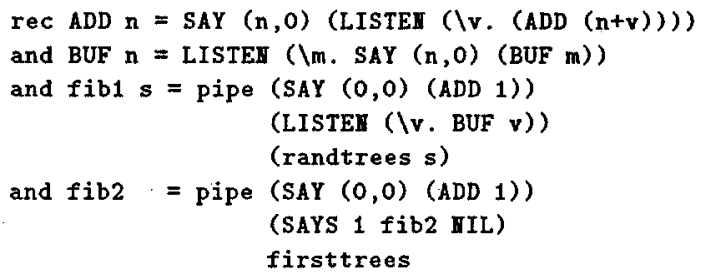

$f i b 1$ is an unprioritised solution. $f i b 2$ is a Kahn network. The output is fed back at priority 1 , and so has to synchronise with the adder's pauses. fib2 is sure to work only with firsttrees. Otherwise, it can get into a loop should the oracle choose the output as speaker when it is not ready.

EXAMPLE 24. [Broadcast sort]

This is a parallelised insertion sort. The input list is made into a process by SAYS and fed into SORTER. The input so far is held in a sorted list, maintained by CELLs each holding a number $n$ and a "link" $l$, the next lower number. The next input number splits exactly one cell into two. BOT and TOP are the end versions of CELL. The output phase is initiated by BOT succeeding in transmitting the head of the sorted list at low priority. Each cell $(l, n)$ transmits $n$ when it hears $l$, at high priority if $l=n$. This last is crucial. Otherwise, the input list $[1 ; 2 ; 2 ; 3]$ would be sorted into PARS [BOT 1; CELL 12 ; CELL 22 ; CELL 23 ; TOP 3] and the output could come out as $[1 ; 2 ; 3 ; 2]$ since both $(2,2)$ and $(2,3)$ are triggered by 2 .

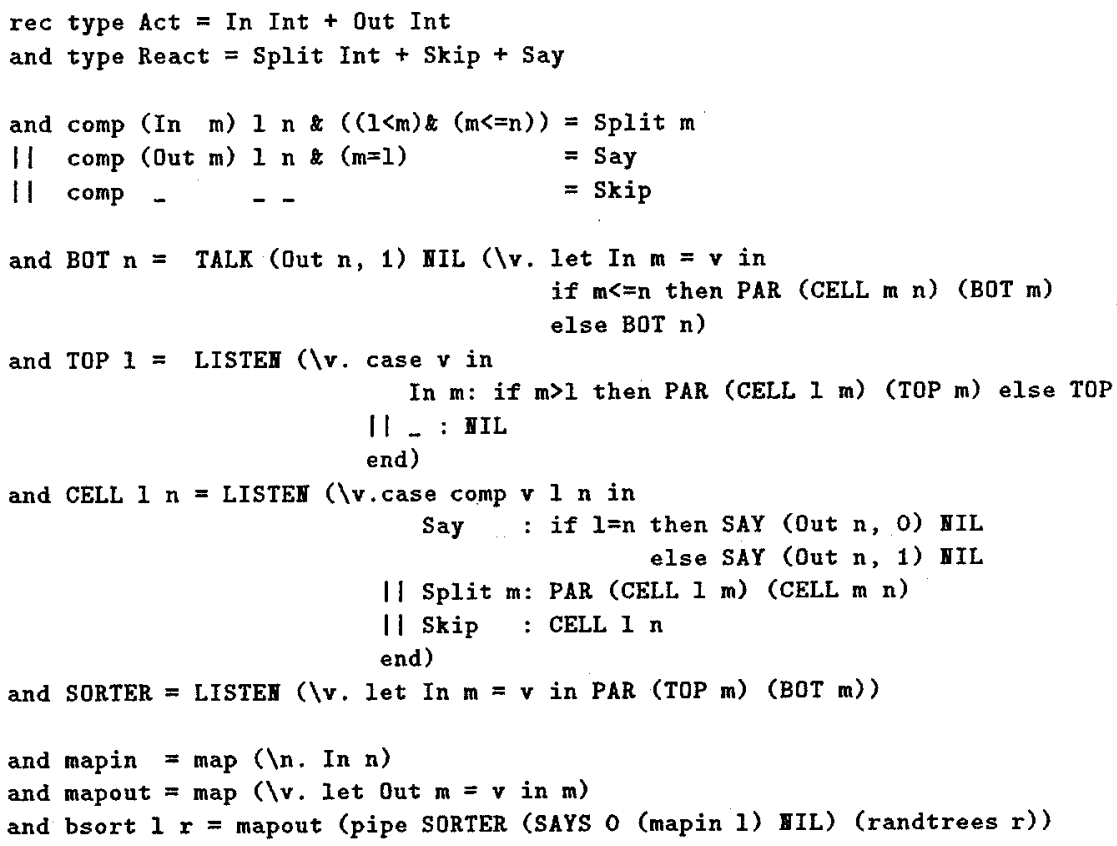

In CBS, duplicates either have to be dropped, or kept track of by a third parameter to CELL. To see how a proof of correctness would go, see [Pra93b]. 
EXAMPLE 25. [Distributed backtrack: The eight queens problem] This program is not limited to queens (redefine the function check) or to any relation between the size of the board and the number of pieces that can be placed.

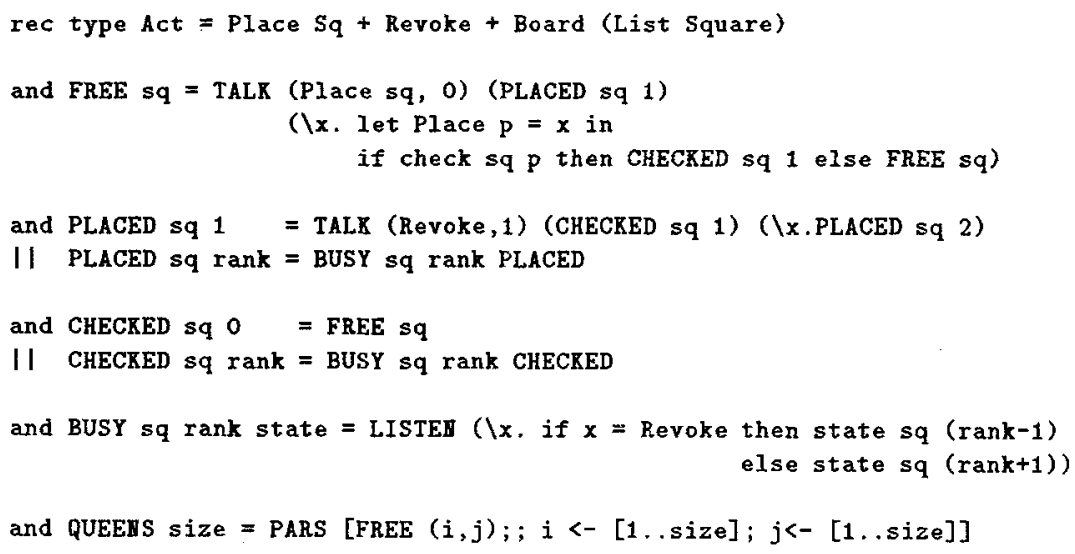

Each square is a process, FREE to start with. Free squares try to grab a piece; one succeeds. As a result, others may become CHECKED and will then await a Revoke by the square that checked them. It will eventually happen that no free square remains, perhaps before the maximum number of pieces have been placed. This is detected by the last placed piece, which succeeds in doing a low priority Revoke. A revoked square pretends it was checked by the previous piece, thus avoiding looping.

The program puts out sequences of Places and Revokes till all solutions have been exhausted. It is equivalent to a sequential one with a stack; PLACED squares say where they are on this stack. Because of the non-determinism, the first solution can often be found very quickly, with few or no Revokes. Finding all solutions takes the same total time no matter how the non-determinism is resolved.

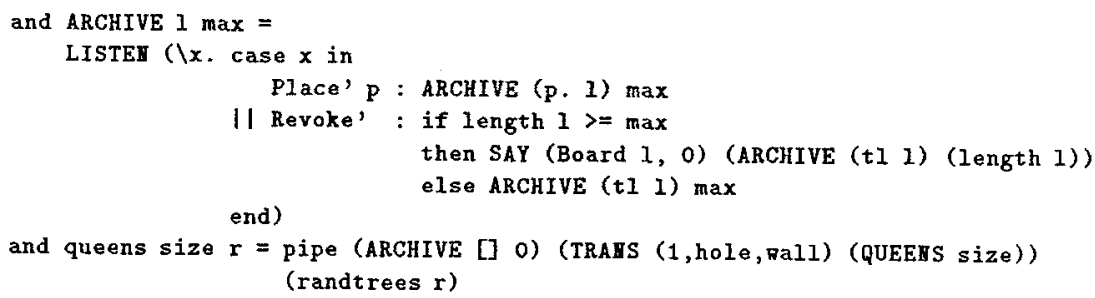

ARCHIVE is one of many possible printer processes. It prints all maximal solutions, and all locally maximal ones prior to the first. It prints at a higher priority to avoid loss of information from QUEENS. Note the use of deprioritisation to avoid adjusting the priorities in QUEENS, and to keep 0 as the highest priority in it.

EXAMPLE 26. [Distributed search: Root of a monotonic function] Given a monotonic function $f:$ Float $\rightarrow$ Float, and a $y$ : Float, binary search is a sequential way to find the $x$ within range such that $f x=y$ to an accuracy given by enough. (If there is no such $x$, the program loops). 
The program below parallelises the search by dividing the range into $\mathrm{n}$ sections. CELL $(k, n)$ computes $f x$, where $x$ is the midpoint of the $k$ th section. When a process converges to $f x$, it reports this value, and range is adjusted. This may result in some processes finding that their $x$ is no longer inrange, in which case they abandon their current computation, and start a fresh one.

This description assumes computations can be interrupted. Otherwise, each round of computation has all processes ready to report, and all but the two nearest the root will find that they have completed a useless computation. So processes come up for communication several times during a computation, which is divided into grain sized bits. Before each round, newst is computed from state (the function $f$ is built into next). If a CELL has nothing to report for that round, it WAITs. If it does have something TOSAY, it will to do so, unless it hears a better value. When no one has anything more to say, they hear a Tick from TRAP, and continue with their computations.

Without priorities, every process would have to report after each round.

To avoid long lists of parameters, several have been built into the program below. A running version should have TOSAY speaking at priority 0 , and deprioritise the array of CELLs.

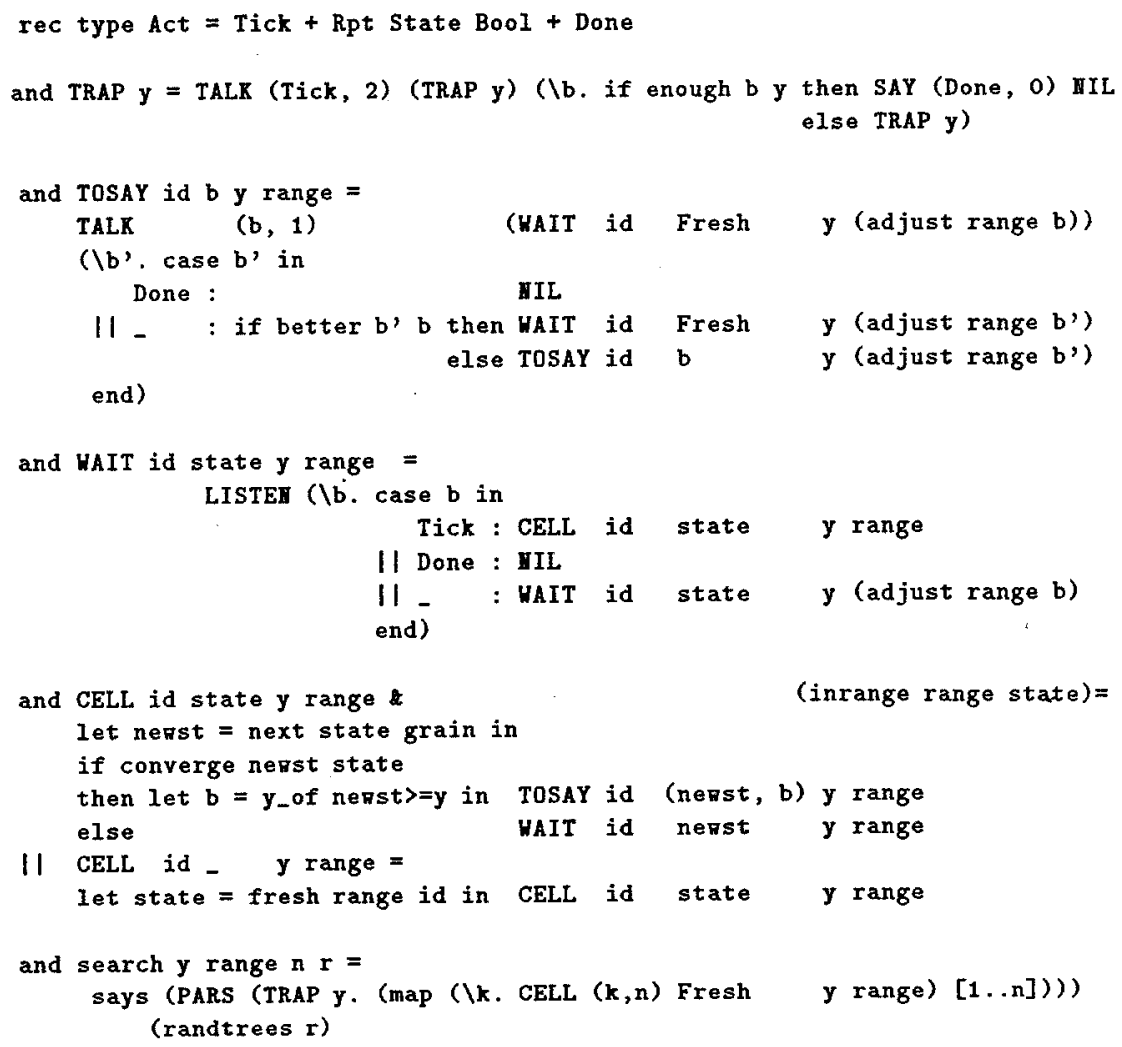


Of the examples in this section, the first two might be called "concurrent" programs, and the rest "parallel". (P)CBS has an implementation on top of a quasiparallel evaluator [RW93], which shows parallelism profiles. This shows that most of the programs here are so fine grain that communication overheads drown the parallelism, at least in this implementation. The CBS formulation is then valuable for its structure rather than parallelism. By contrast, the distributed search above allows significant parallelism and meaningful experiments in optimisation and load balancing by varying grain and $\mathbf{n}$.

\section{IMPLEMENTATION; INTEgRATION OF EVALUATION AND COMMUNICATION}

[Pra93c] presents an extended calculus with both evaluation and communication transitions that is the basis for an implementation by a simulator in a functional language. Evaluation is borrowed from the host language, and accounts for recursion and conditionals. All of this can be extended to PCBS. A study of the implementation, including a proof that the concrete treatment of $\tau$ 's is equivalent to the abstract one, is left to a forthcoming report.

\section{Conclusions}

Priorities can be added easily to CBS, and significantly extend its power.

The transition system for PCBS yields only those transitions that can actually take place. It differs from that of CBS primarily in the side conditions for the prefix rules. The only other material change is the possibility of deprioritisation.

PCBS is simple because only autonomous actions need be considered when deciding which process should act next. These actions are distinct ones to which it is meaningful to assign priorities. By contrast, it is precisely the fact that there is only one autonomous action in CCS that makes it difficult to add priorities to it. Encoding a negative premise about transmission as a positive one about reception compacts the system further.

Strong and weak bisimulations in PCBS are congruences, and yield observationally meaningful equivalences. Putting deprioritisation aside, only only law distinguishes PCBS from CBS: outranked output guards in a sum cannot be used. This corresponds to a similar law in [CH88]. Interestingly, neither the expansion theorem nor the law of distribution of translation over / need change.

Priorities make a useful addition to the CBS paradigm. They provide atomic sequences (detecting termination, absence of response, etc.), preemption of default actions by exceptions (or interrupts), and a means of doing some actions immediately. Proofs were dropped in favour of more examples, but it should be clear that the usual techniques of process calculus are applicable.

[Pra93b, Pra93c] suggest that CBS could be a practical programming language. This paper records a further step, experiments with a parallelism profiler.

\section{Related AND FUtURE WORK}

[Pra93a] develops a Timed CBS. It shows that the same aspects of broadcast communication are again useful, and that PCBS can be derived from Timed CBS. Most of Timed CBS can be derived from PCBS. [Jef92] suggests that a (discrete) timed calculus can be derived from a prioritised calculus; one relevant aspect here is that PCBS appears to extend to dense priorities with no change. 
Adding probabilities to CCS (CBS) is difficult (easy) for the same reasons as adding priorities is.

Future work for CBS includes the development of a proof system, further development of implementations, and comparison with such systems as LINDA [CG89], GAMMA [BM91] and I/O automata [LT87]. These will be extended to PCBS.

Statecharts [Har87] and ESTEREL [BG92] both use broadcast communication, and deal with interrupts and timeouts. However, the models are different enough that comparison is difficult. ESTEREL, for example, allows multiple signals to be broadcast șimultaneously, and the receiver chooses which to act on.

Acknowledgements. I thank members of the concurrency and functional programming groups at Chalmers for support and encouragement.

\section{REFERENCES}

[AJ93] Lennart Augustsson and Thomas Johnsson. Lazy ML user's manual. Technical report, Department of Computer Science, Chalmers University of Technology, 1993.

[BA90] M. Ben-Ari. Principles of Concurrent and Distributed Programming. Prentice-Hall, 1990.

[BBK85] J. C. M. Baeten, J. A. Bergstra, and. J. W. Klop. Syntax and defining equations for an interrupt mechanism in process algebra. Technical Report CSR8503, Centre for Mathematics and Computer Science, Amsterdam, 1985.

[BG92] Gérard Berry and Georges Gonthier. The ESTEREL synchronous programming language: Design, semantics, implementation. Science of Computer Programming, 19, 1992.

[BM91] J.-P. Banâtre and D. Le Metayer, editors. Research Directions in High-Level Parallel Programming Languages. Springer Verlag LNCS 574, 1991.

[Bur88] F. W. Burton. Nondeterminism with referential transparency in functional languages. The Computer Journal, 31(3):243-247, 1988.

[CC93] Linda Christoff and Ivan Christoff. Observational equivalence for processes with priorities. Technical report, Dept. of Computer Systems, Uppsala Univ., 1993.

[CG89] Nicholas Carriero and David Gelernter. Linda in context. Communications of the ACM, 32(4):444-458, April 1989.

[CH88] Rance Cleaveland and Matthew Hennessy. Priorities in process algebras. In Symposium on Logic in Computer Science. IEEE, 1988.

[CW91] Juanito Camilleri and Glynn Winskel. CCS with priority choice. In Symposium on Logic in Computer Science. IEEE, 1991.

[Har87] David Harel. Statecharts: A visual formalism for complex systems. Science of Computer Programming, 8, 1987.

[Jef92] Alan Jeffrey. Translating timed process algebra into prioritised process algebra. In $\mathrm{Ni}$ jmegen Symposium on Real-Time and Fault-Tolerant Systems, 1992.

[LT87] Nancy Lynch and Mark Tuttle. Hierarchical correctness proofs for distributed algorithms. Technical Report MIT/LCS/TR-387, Laboratory for Computer Science, Massachusetts Institute of Teclinology, 1987.

[Mil89] Robin Milner. Communication and Concurrency. Prentice Hall, 1989.

[Pra91] K. V. S. Prasad. A calculus of broadcasting systems. In TAPSOFT'91 Volume 1: CAAP, April 1991. Springer Verlag LNCS 493.

[Pra93a] K. V. S. Prasad. Broadcasting in time. Technical report, Department of Computer Science, Chalmers University of Techmology, 1993.

[Pra93b] K. V. S. Prasad. A calculus of value broadcasts. In PARLE'93, June 1993. Springer Verlag LNCS 694.

[Pra93c] K. V. S. Prasad. Programming with broadcasts. In CONCUR'93, August 1993. Springer Verlag LNCS 715.

[RW93] C. Runciman and D. Wakeling. Profiling parallelism. Internal report, Department of Computer Science, University of York, 1993. 